\title{
The Multidimensional Compressed Storage of Upper-half banded matrix
}

\author{
Yongyan $\mathrm{Hou}^{\star 1, \mathrm{a}}$, Zhengping $\mathrm{Zhu}^{2}$, Lanqing $\mathrm{Wu}^{3}$ \\ ${ }^{1}$ Zhixing College of Northwest Normal University. No.20 Changxin Road, Anning District, Lanzhou City, \\ Gansu Province,730070, P.R.China; \\ ${ }^{2}$ Zhengping Zhu(Corresponding Author) School of Electronics and Information Engineering, Lanzhou City \\ University. No.11 Jiefang Road, Anning District, Lanzhou City, Gansu Province,730070, P.R.China \\ ${ }^{3}$ School of Electronics and Information Engineering, Lanzhou City University. No.11 Jiefang Road, Anning \\ District, Lanzhou City, Gansu Province,730070, P.R.China \\ aren_zhiguo@qq.com
}

Keywords: Multidimensional; Compressed Storage; Upper-half banded matrix

\begin{abstract}
Matrix is commonly used in scientific computing and engineering calculation. We are not concerned about data itself in data-structures, but how to store the elements in it, and make all the operations run effectively. The main task of the compressed storage is make more of the same nonzero-elements share the same storage unit according the distribution of elements, at the same time lots of zero-elements don't allocate storage space. In this article, we discussed the multidimensional compressed storage of upper-half banded matrix, and obtained the row-major compressed storage address mapping functions and columnmajor compressed storage address mapping functions for the first time. Also, the two kinds of compressed storages have high compression storage ratio. We hope the conclusion can provide the basic data storage theory for engineering calculation and scientific computing.
\end{abstract}

\section{DEFINITION OF UPPER-HALF BANDED MATRIX}

Matrix is a mathematical object, commonly used in scientific computing and engineering calculation. We are not concerned about data value or type in data-structures, but how to store the elements in the matrix. In computer programming we often use a two-dimensional array to store the elements of the matrix. If we adopt two-dimensional array for the matrix, we can access the data elements in the matrix randomly, thus the operations for the matrix can easily realizing. But, when there are lots of zero-elements in the matrix and have regular distribution, if we still use a two-dimensional array to store the matrix elements, The particular elements will consume large numbers of storage space. If a matrix is a high order matrix, the storage method is not only waste storage space, but also takes a lot of time for invalid computation, it is obviously not desirable. In order to reduce the storage space, we need to compress storage for such matrix.

The main purpose of the compressed storage is to make more of the same nonzero-elements share the same storage unit according the distribution of the element in the matrix. At the same time, the zeroelements don't allocate storage space. In this article, we discussed the compressed storage problems of upper-half banded matrix, and obtained the corresponding storage address mapping functions.

The upper-half banded matrix is evolved from the banded matrix. The elements come from the banded matrix diagonal line and upper part only. The elements along the diagonal direction of the matrix regular distribution in the one side of the diagonal. In a N-order matrix $\mathrm{M}$, if there exists a maximum positive number $\mathrm{W}$, The $m_{i, j}$ is a matrix element, when it meets $\theta_{\mathrm{j}}-\mathrm{i} \leq \mathrm{W}-1, m_{i, j}$ is an ordinary integer, the rest elements are 0 or a constant integer. The $M$ is called upper-half banded matrix. The integer $\mathrm{m}$ is called 
bandwidth. If the order is $\mathrm{N}$, the bandwidth is $\mathrm{W}$, the data elements in the half band matrix $\mathrm{M}$ compressed into a multidimensional D. By the calculation, the size of storage space for dimensional $\mathrm{D}$ is $(\mathrm{W} \times \mathrm{N})$.

\section{ROW-MAJOR COMPRESSED STORAGE OF UPPER-HALF BANDED MATRIX}

When $\mathrm{N}=5, \mathrm{~W}=2$, the corresponding upper-half banded matrix $\mathrm{M}$ can be stored into a multidimensional storage space D. As shown in figure 1.

$$
M=\left[\begin{array}{ccccc}
a_{11} & a_{12} & 0 & 0 & 0 \\
0 & a_{22} & a_{23} & 0 & 0 \\
0 & 0 & a_{33} & a_{34} & 0 \\
0 & 0 & 0 & a_{44} & a_{45} \\
0 & 0 & 0 & 0 & a_{55}
\end{array}\right] \Rightarrow D=\left[\begin{array}{cc}
a_{11} & a_{12} \\
a_{22} & a_{23} \\
a_{33} & a_{34} \\
a_{44} & a_{45} \\
a_{55} & 0
\end{array}\right]
$$

Figure. 1 The compression storage results when $\mathrm{N}=5, \mathrm{~W}=2$

As shown in figure 2 . When $\mathrm{N}=5, \mathrm{~W}=3$, the upper-half banded matrix $\mathrm{M}$ can be stored into a twodimensional array $\mathrm{D}$ through the above method.

$$
M=\left[\begin{array}{ccccc}
a_{11} & a_{12} & a_{13} & 0 & 0 \\
0 & a_{22} & a_{23} & a_{24} & 0 \\
0 & 0 & a_{33} & a_{34} & a_{35} \\
0 & 0 & 0 & a_{44} & a_{45} \\
0 & 0 & 0 & 0 & a_{55}
\end{array}\right] \Rightarrow D=\left[\begin{array}{ccc}
a_{11} & a_{12} & a_{13} \\
a_{22} & a_{23} & a_{24} \\
a_{33} & a_{34} & a_{35} \\
a_{44} & a_{45} & 0 \\
a_{55} & 0 & 0
\end{array}\right]
$$

Figure. 2 the compression storage results when $\mathrm{N}=5, \mathrm{~W}=3$

We can found obviously, the diagonal line elements are carried to the right of the two-dimensional array D, the lowest diagonal line elements are carried to the left of array D. The rest of the elements are stored in the array D by this method. The row index of element $m_{i, j}$ does not change, the column index is changed to $(\mathrm{j}-\mathrm{i}+1)$. Then get the mapping relationship $\mathrm{f}(\mathrm{i}, \mathrm{j})$ from $\mathrm{m}_{\mathrm{i}, \mathrm{j}}$ to $\mathrm{d}_{\mathrm{i}^{\prime} \mathrm{j}}{ }^{\prime}$ (formula 1$)$ :

$$
f(i, j)=\left\{\begin{array}{c}
i^{\prime}=i \\
j^{\prime}=j-i+1
\end{array}\right.
$$

\section{COLUMN-MAJOR COMPRESSED STORAGE OF UPPER-HALF BANDED MATRIX}

When $\mathrm{N}=5, \mathrm{~W}=2$, the corresponding upper-half banded matrix $\mathrm{M}$ can be stored into a twodimensional array D by column-major compression method. As shown in figure 3.

$$
M=\left[\begin{array}{ccccc}
a_{11} & a_{12} & 0 & 0 & 0 \\
0 & a_{22} & a_{23} & 0 & 0 \\
0 & 0 & a_{33} & a_{34} & 0 \\
0 & 0 & 0 & a_{44} & a_{45} \\
0 & 0 & 0 & 0 & a_{55}
\end{array}\right] \Rightarrow \mathrm{D}=\left[\begin{array}{ccccc}
0 & a_{12} & a_{23} & a_{34} & a_{45} \\
a_{11} & a_{22} & a_{33} & a_{44} & a_{45}
\end{array}\right]
$$

Figure. 3 the compression storage results when $\mathrm{N}=5, \mathrm{~W}=2$

As shown in figure 4 . When $\mathrm{N}=5, \mathrm{~W}=3$, the corresponding upper-half banded matrix $\mathrm{M}$ can be stored into a two-dimensional array $\mathrm{D}$ through above method. 


$$
M=\left[\begin{array}{ccccc}
a_{11} & a_{12} & a_{13} & 0 & 0 \\
0 & a_{22} & a_{23} & a_{24} & 0 \\
0 & 0 & a_{33} & a_{34} & a_{35} \\
0 & 0 & 0 & a_{44} & a_{45} \\
0 & 0 & 0 & 0 & a_{55}
\end{array}\right] \Rightarrow D=\left[\begin{array}{ccccc}
0 & 0 & a_{13} & a_{24} & a_{35} \\
0 & a_{12} & a_{23} & a_{34} & a_{45} \\
a_{11} & a_{22} & a_{33} & a_{44} & a_{55}
\end{array}\right]
$$

Figure. 4 the compression storage results when $\mathrm{N}=5, \mathrm{~W}=3$

The above figure can be found as a conversion: The sub array of the upper-half banded matrix $\mathrm{M}$ are carried out to the left, so as to achieve the compression for storage the upper-half banded matrix M.

We can found, the diagonal line elements are carried to the upper of the two-dimensional array D. The lowest diagonal line elements are carried to the lowest of array $\mathrm{D}$. The rest of the elements are stored in the array $\mathrm{D}$ by this method. The column index of element $\mathrm{m}_{\mathrm{i}, \mathrm{j}}$ does not change, the raw index is changed to (i$\mathrm{j}+\mathrm{m}$ ). Then we can get the mapping relationship $\mathrm{f}(\mathrm{i}, \mathrm{j})$ from $\mathrm{m}_{\mathrm{i}, \mathrm{j}}$ to $\mathrm{d}_{\mathrm{i}^{\prime} \mathrm{j}^{\prime}}$ (formula 2 ):

$$
f(i, j)=\left\{\begin{array}{c}
i^{\prime}=i \\
j^{\prime}=j-i+W
\end{array}\right.
$$

\section{CONCLUSION}

In the article, we discussed the multidimensional compressed storage problems of upper-half banded matrix. Then studied the row-major and column-major compressed storage problems, obtained the rowmajor compressed storage address mapping functions of the multidimensional compressed storage (formula.1), also obtained the column-major compressed storage address mapping functions of the multidimensional compressed storage about it (formula.2). In an N-order matrix with W-band width, the data elements can be stored into a multidimensional array, its compression storage ratio can be obtained $\mathrm{CSR}=\left(1-(\mathrm{W} \times \mathrm{N}) / \mathrm{n}^{2}\right) \times 100 \%$. In a 100 -order square matrix of 5-band width, the compression storage ratio CSR is: CSR $=\left(1-(5 * 100) / 100^{2}\right) * 100 \%=95 \%$. So we found these two kinds of compressed storages have high compression storage ratio. We hope these conclusions can provide the basic theory of compression storage for the scientific computing.

Supported by Association of Fundamental Computing Education in Chinese Universities (AFCECU:2016084);Supported by the Education and Teaching Research Foundation (2013-JY-25) and Doc's Foundation of Lanzhou City University (LZCU-BS2013-12; LZCU-BS2013-09).

\section{REFERENCES}

1. Thomas H.Cormen,Charles E.Leiserson,Ronald L.Rivest,Clifford Stein. Introduction to Algorithm, the third edition. The MIT Press,2009.

2. Alfred V.Aho,John E.Hopcroft,and Jeffrey D.Ullman.Data structures and Algorithms. AddisonWesley, 1983.

3. Zhiguo Ren, Data Structure(C Language Description), Science Press, Peking China, 2016.

4. Donald E.Knuth.Fundamental Algorithms,volume 1 of The Art of Computer Programming. AddisonWesley, 1968.Third edition,1997.

5. Donald E.Knuth.Seminumerical Algorithms,volume 2 of The Art of Computer Programming. Addison- Wesley,1969.Third edition,1997.

6. Don Coppersmith and Shmuel Winograd.Matrix Multiplication via arithmetic progression. Journal of Symbolic Computation,9(3):251-280,1990.

7. T.C.Hu and M.T.Shing.Computation of Matrix chian products.Part 1,SIAM Journal on Computing, 11(2):362-373,1982. 
8. Mark Allen Weiss.Data Structures and Algorithm analysis in Java. Addison-Wesley,third edition,2007.

9. Ludeña-Choez, J., \& Gallardo-Antolín, A. (2016). Acoustic event classification using spectral band selection and non-negative matrix factorization-based features. Expert Systems with Applications,46(C), 77-86.

10. Osipov, \& Andrey. (2016). A study of resolvent set for a class of bandoperators with matrix elements. Concrete Operators, 3(1), 85-93. 